\title{
Current and Future Systemic Therapies in Biliary Tract Cancer
}

\author{
Arndt Vogel Anna Saborowski \\ Department of Gastroenterology, Hepatology and Endocrinology, Medical School Hannover, Hannover, Germany
}

\section{Keywords}

Cholangiocarcinoma - Gall bladder cancer - Chemotherapy .

Precision oncology $\cdot$ Molecular therapies

\begin{abstract}
Background: Despite an increasing incidence, biliary tumors are still considered a rare tumor entity. Due to an often long clinically inapparent course and a lack of early detection strategies, surgical resection is often not possible at the time of diagnosis. Since 2010, chemotherapy with gemcitabine and cisplatin is considered the standard of care in the palliative situation. Only recently, first studies have been published or initiated that expand the treatment options in the first line and, for the first time, also suggest valid systemic approaches in the second line. Summary: Molecularly targeted therapies in selected patient subgroups are rapidly changing the field. In addition to IDH1 mutations and FGFR2 fusions in patients with intrahepatic tumors, the therapeutic relevance of rare but targetable alterations such as HER2/neu amplification, NTRK fusions, or BRAF mutations should be considered in patients with biliary tract cancers. Key Message: The current study landscape clearly shows that precision medicine will play an important role in the therapy of biliary malignancies and underlines the importance of early tumor genetic diagnostics. In this article we provide an overview of systemic therapy concepts in the adjuvant and palliative setting.
\end{abstract}

(C) 2021 The Author(s)

Published by S. Karger AG, Basel

\section{Introduction}

Cancers of the biliary system (BTC) are highly aggressive tumors that either originate within the liver (intrahepatic cholangiocarcinoma [iCCA]), in the perihilar or dis- tal bile ducts (perihilar or distal CCA), or in the gallbladder [1]. In most countries, CCA is considered a "rare" cancer with incidence rates below $6 / 100,000$. However, due to the demographic distribution of different risk factors and probably influenced by ethnic factors, the incidence of CCA ranges from $0.1 / 100,000$ in Australia to more than $110 / 100,000$ in northeast Thailand. In Europe, most biliary tumors occur sporadically after the age of 50 , with a slight predominance of male patients. Risk factors include obesity, viral hepatitis B and C, primarily sclerosing cholangitis, and, for gallbladder carcinoma, gallstones. In Asian countries, infestation with parasitic liver flukes is considered an important risk factor. As the only potentially curative therapy, surgical resection should be offered to patients who are diagnosed at an early stage. However, due to the late manifestation of clinical symptoms, most patients suffer from locally advanced or metastatic disease at the time of diagnosis, and even after successful resection early recurrence is frequent. Therefore, most patients are eventually bound to receive palliative treatments. Unfortunately, BTCs are relatively chemoresistant tumors, and the median overall survival (mOS) of 11-13 months under systemic palliative therapy with gemcitabine and cisplatin highlights the urgent need for new therapeutic approaches $[2,3]$.

In the following, we provide an overview of the treatment strategies that are currently available, or are under clinical consideration, for the systemic treatment of biliary cancers.

\section{Adjuvant Therapy}

The high 3-year rate of recurrence that can be up to $80 \%[4,5]$ after curatively intended resection has led to an intensive discussion about the importance of adjuvant 
therapy concepts. Until 2017, the use of adjuvant treatment was based on meta-analyses from mostly small retrospective phase II studies, which suggested that specifically two high-risk populations benefit from postoperative chemotherapy in terms of mOS: patients with nodal-positive disease and following $\mathrm{R} 1$ resection $[6,7]$. A similar trend was also observed in our own patient population in a retrospective matched pair analysis [8].

Recently, three prospective randomized clinical trials have been published in which different adjuvant chemotherapy concepts were investigated: the French PRODIGE-12, the Japanese BCAT, and the British BILCAP study. The French PRODIGE-12/ACCORD-18 study, first published in 2017, showed no benefit of adjuvant gemcitabine/oxaliplatin (GemOx) compared to observation alone in patients with CCA and GBC in terms of RFS (30.4 months vs. 18.5 months in the observation arm; HR 0.88; 95\% CI $0.62-1.25 ; p=0.48$ ) [9]. Similarly, the Japanese BCAT study evaluating gemcitabine as a monotherapy in the adjuvant arm in a 225-patient population was negative for RFS (median 36.0 vs. 39.9 months; HR 0.93 ; $95 \%$ CI $0.66-1.32 ; p=0.693)$ and OS (62.3 vs. 63.8 months; HR $1.01 ; 95 \%$ CI $0.70-1.45 ; p=0.964$ ) [10].

The BILCAP study was conducted in the UK over a period of 11 years and is the largest study involving both CCC and GBC patients. The study did not meet its primary endpoint in terms of mOS in the ITT population; however, a pre-specified ITT sensitivity analysis adjusted for prognostic factors (lymphonodal status, tumor grading, and sex) revealed a significantly longer mOS in the capecitabine arm compared to the observation arm (53 months vs. 36 months; HR 0.75 ; 95\% CI $0.58-0.97$; $p=$ 0.028) [11]. In the ITT analysis, there was also a trend for a longer median RFS in the capecitabine arm compared to observation (24.4 months vs. 17.5 months; HR 0.81; $95 \% \mathrm{Cl} 0.63-1.04 ; p=0.097)$. Based on the available results, the control arm was adjusted in the currently recruiting German ACTICCA study (NCT02170090): gemcitabine and cisplatin are now compared to capecitabine (Xeloda) rather than to observation.

Despite the lack of a formally positive prospective study, we believe that, based on the published data, adjuvant treatment with capecitabine for 6 months should be discussed with patients following resection. To what extent an intensification of the adjuvant therapy with gemcitabine and cisplatin is necessary or meaningful cannot yet be decided. Adjuvant and/or neoadjuvant therapy concepts may be especially beneficial in patients with a high risk of very early relapse. A recent study showed that very early recurrence within 6 months after surgery occurs in almost $25 \%$ of iCCA patients and is associated with a dismal mOS of 13.8 months and a 5 -year OS of $8.9 \%$, compared to 59.7 months and $49.8 \%$ $(p<0.001)$ in patients without very early recurrence [5].
Not surprisingly, tumor size (OR 1.11), number of tumors (OR 1.36), microvascular invasion (OR 1.55), N1 or Nx status (OR 1.94), and R1 resection (OR 2.14) were associated with a higher probability of early recurrence. Complementing these clinical parameters, the detection of circulating tumor DNA will most likely help in the future to identify patients with a high risk of relapse, who should receive either adjuvant therapy or at least a close follow-up.

\section{Palliative Therapy}

\section{First Line}

The ABC-02 phase-III study, published in 2010, and the Japanese BT22 phase II study established the combination of gemcitabine and cisplatin in the first-line treatment of BTC $[3,12,13]$. As an alternative platinum-based regimen, the combination of gemcitabine with oxaliplatin has been investigated in several phase II trials and showed comparable efficacy and safety [14]. In addition, a South Korean phase III trial recently confirmed the non-inferiority of oxaliplatin and capecitabine (XelOx) compared to GemOx [15]. Similar to pancreatic cancer, a phase II trial tested gemcitabine/nab-paclitaxel in the first-line setting and showed comparable efficacy to GemCis, although a direct randomized comparison of the two therapies has not yet been performed [16]. The phase Ib ABC-08 trial investigated cisplatin in combination with NUC-1031, a phosphoramidate variant of gemcitabine that bypasses basic resistance mechanisms to the nucleoside analogue. Based on a remarkable ORR of $63.6 \%$, the combination is currently pursued in a phase III trial (NCT04163900) [17].

The extent to which a further intensification of chemotherapy with a triplet is possible and useful has been addressed in various studies, including a trial in which nabpaclitaxel was combined with cisplatin and gemcitabine [18]. Despite a toxicity-related reduction of the gemcitabine dose, the study reached a mPFS of 11.8 months (95\% CI 6.0-15.6 months) with a DCR of $84 \%$, and an impressive mOS of 19.2 months. This combination is currently being compared directly with the standard of care in the SWOG-1815 phase III study (NCT03768414). In contrast, the results of the French phase II PRODIGE38 study, which investigated the efficacy of modified FOLFIRINOX compared to the standard of care (GemCis) in first line were disappointing. After a median follow-up of 21 months, the primary endpoint was not met in the mFOLFIRINOX arm with a 6-month PFS rate of $44.6 \%$ (90\% CI 35.7-53.7) [19]. The median PFS (ITT) was 6.2 months (95\% CI 5.5-7.8) in the mFOLFIRINOX group compared to 7.4 months (95\% CI 5.6-8.7) in the GemCis group, and the mOS was 11.7 months (95\% CI 9.5-14.2) 
versus 14.3 months in the standard therapy arm (95\% CI 11.3-16.5). Based on these data, this triplet will not be further investigated in the originally planned phase III study.

In all studies on BTC, the composition of the trial populations should be taken into account, as the prognostic significance of tumor localization is becoming increasingly apparent. A recent combined post-hoc analysis of the ABC-01/02/03 studies revealed that the mOS of patients with intrahepatic CCAs was approximately 4 months longer than that of patients with biliary tumors that originated in other locations, suggesting a more prolonged course of disease especially in patients with liverlimited iCCA [20].

\section{Second Line}

Until recently, the efficacy of second-line therapy in patients with advanced biliary tumors was not proven. In a systematic analysis involving nearly 700 patients from 14 phase II studies and several retrospective analyses and case reports, the mPFS and mOS were 3.2 and 7.2 months, respectively, after the start of second-line chemotherapy [21].

The British ABC-06 study presented at ASCO 2019 was the first phase III study that evaluated a second-line approach after first-line therapy with GemCis: mFOLFOX led to a $15 \%$ improvement of both the 6- and 12-month survival in ECOG 0/1 patients compared to observation. The chemotherapy arm had a moderately improved mOS of 6.2 months, compared to 5.3 months in the control arm $(\mathrm{HR}=0.69 ; 95 \%$ CI $0.5-0.97 ; p=0.031)$. As a note of caution it should be mentioned that, according to the recommendations in the United Kingdom, the majority of patients receive a predetermined number of treatments in first line (8 cycles of GemCis), whereas patients in most other countries, including Germany, are more frequently treated until disease progression. The extent to which oxaliplatin-based chemotherapy after cisplatin actually leads to a clinically significant prolongation of survival in patients who progressed under GemCis is not proven. In a Dutch single-arm phase II trial, promising results were shown for the use of FOLFIRINOX in fit patients with a DCR of $67 \%$, a PFS of 6.2 months (95\% CI 3.0-9.1), and a mOS of 10.7 months (95\% CI 5.5-15.4) [22]. The mOS for sequential GemCis, followed by FOLFIRINOX, was 18.5 months (95\% CI 13.5-21.4). In daily clinical life, an escalation from doubled to triplet chemotherapy is, however, highly unlikely, and the triplet may - if at all - be considered in the first-line setting. In Germany, the AIO NALIRICC study currently investigates the efficacy of Nal-IRI in combination with 5-FU versus 5-FU alone (NCT03043547) in the second-line setting.

In summary, based on the currently available data, second-line chemotherapy should be offered to fit patients who progress on first-line therapy. We usually recom- mend a 5-FU-based combination with irinotecan or oxaliplatin, alternatively 5-FU or capecitabine monotherapy may be considered.

\section{Targeted Therapies}

To date, all studies that employed molecular therapies in unselected BTC cohorts were negative, including antiangiogenetic agents $[2,23]$. Even in RAS WT patients, epidermal growth factor receptor (EGFR)-directed therapy failed to show added value over the chemotherapeutic backbone GemCis [24]. In recent years, a detailed characterization of the molecular landscape of biliary tumors has been conducted and revealed that BTCs are a genetically heterogeneous group of malignancies. However, these studies also identified a subset of recurrent and targetable alterations. Especially in patients with iCCA, nearly $40 \%$ of patients harbor genetic alterations which are potential targets for precision medicine [25-27]. If tumor tissue is available, we recommend that molecular analysis should be performed preferentially already before or during first-line therapy in order to evaluate options for second and higher lines early during the course of treatment. In particular, the following genetic alterations should be considered:

\section{IDH $1 / 2$}

The neomorphic activity of the mutant IDH1/2 enzymes results in an increased production of the oncometabolite 2-hydroxyglutarate (2-HG) from a ketoglutarate. In preclinical models, $2-\mathrm{HG}$ leads to a number of protumorigenic changes in multiple tumors including BTC. The most common, clinically relevant mutations in IDH1 and -2 occur at amino acid positions 132 (R132) and 172 (R172), respectively. These mutations are found in about $10-20 \%$ of iCCA patients but are likely without prognostic significance.

Ivosidenib is an oral inhibitor of the mutant IDH1 enzyme and thus far the only targeted agent that has successfully completed a phase III trial in CCA. In the ClarIDHy phase III study, the efficacy of ivosidenib was evaluated in comparison to placebo in 185 IDH1 mutant CCA patients who progressed on first-line therapy [28]. $\mathrm{Pa}$ tients were randomized to ivosidenib or placebo at a 2:1 ratio and crossover to ivosidenib was permitted in the placebo arm after progression. The primary endpoint an improvement in PFS - was achieved with an impressive HR of 0.37 (95\% CI $0.25-0.54$; $p<0.001)$ but with a mPFS of only 2.7 months for ivosidenib compared to 1.4 months for placebo. Regardless of the minor absolute PFS difference and the low radiological response rate of $2.4 \%$, PFS rates at 6 and 12 months were clinically relevant $(32.0$ and $21.9 \%$ in the ivosidenib arm at 6 and 12 months, re- 
spectively; $0 \%$ at both timepoints in the placebo group). Due to the crossover design, the influence on the OS can only be assessed to a limited extent. Following statistical adjustment for the crossover design, mOS of 10.8 months under ivosidenib was significantly improved compared to the mOS of 6 months in the placebo arm (HR 0.46; $p=$ 0.0008). Currently, however, FDA or EMA approval has not yet been filed for ivosidenib.

\section{FGFR2}

FGF receptor alterations in malignant tumors include amplifications and mutations as well as oncogenic fusions. These FGFR2 fusions occur in up to $15 \%$ of iCCA patients and are caused by a translocation between the $5^{\prime}$ portion of FGFR 2 and the $3^{\prime}$ end of one of more than 150 fusion partners identified thus far. While the FGFR2 portion contains the intact extracellular and kinase domains, the fusion partners are characterized by a protein dimerization domain, which leads to constitutive dimerization and ligand-independent kinase activation [29].

To date, several phase II studies in patients with iCCA and FGFR2 fusions have revealed a very consistent efficacy of FGFR-targeted therapies in higher lines with a response rate between 20 and $36 \%$ [30, 31]. The FIGHT-202 trial investigated the efficacy of the FGFR inhibitor pemigatinib in progressed fusion-positive iCCA patients and was the largest study conducted in this genetically selected population thus far. Disease control was $85.1 \%$ with an ORR of $36 \%$, and an impressive mPFS and mOS of 9.2 and 15 months, respectively [32]. Based on these results, pemigatinib has received FDA approval for pretreated CCA patients with FGFR2 fusions.

Currently, several FGFR inhibitors have been entered into phase III trials. Since early 2019 , the randomized trial "PROOF" has been recruiting patients in the first line for treatment with infigratinib versus GemCis (and possible crossover into the infigratinib arm) [33]. In addition, pemigatinib is further developed in first line in comparison to standard of care (GemCis) in the FIGHT-302 study (NCT03656536) [34], whereas derazantinib is evaluatedintheFIDES-01 studyin secondline(NCT03230318).

In summary, it appears highly likely that FGFR2 inhibitors will soon become an integral component of the standard systemic therapy for FGFR2 fusion-positive iCCAs also in Europe. Considering the imminent therapeutic relevance of FGFR2 fusions, it appears mandatory that all patients with iCCA are offered genetic testing that is capable of detecting FGFR2 fusions independent of the fusion partner.

\section{HER2}

In an increasing number of solid tumors, HER2/neu (ERBB2) is being recognized as a predictive biomarker and promising target for molecular therapy [35]. While genetic amplification is the most frequent HER2 alteration, activating HER2 mutations are particularly common in biliary tumors. In CCA, HER2 alterations occur in approximately $8-10 \%$ of patients, and nearly $40 \%$ of these are classified as HER2 mutations. In patients with gallbladder carcinoma, the proportion of HER2 amplifications/over-expression is estimated to exceed $10 \%$ [36], and, in addition, almost $10 \%$ of cases harbor active mutations in ERBB2 or ERBB3 (11\%) [37].

The recently published MyPathway basket study investigated the efficacy of dual receptor inhibition using trastuzumab and pertuzumab in heavily pre-treated patients with various solid tumors. In this study, disease control was achieved in 5 out of 7 patients with HER2amplified CCA and in 1 out of 3 patients with HER2-mutated CCA $[38,39]$.

The SUMMIT phase II basket study specifically investigated the response to targeted therapy in patients with HER2- and HER3-mutated, but not -amplified, tumors. By the beginning of 2019, a total of 19 patients with HER2mutant biliary tract tumors had received the pan-HER kinase inhibitor neratinib in higher therapy lines. Treatment was well tolerated with an ORR of 10.5\% (95\% CI 1.3-33.1) and a median PFS of 1.8 months (95\% CI 1.03.7) [40]. Overall, these results suggest that although selected patients seem to benefit, the response in HER2mutant CCA patients is inferior to the response observed in HER2-amplified tumors [38, 39].

Due to the small number and frequently heavily pretreated patients, data on chemotherapy-free, HER2-directed therapies need to be interpreted with caution. Nevertheless, the available information overall supports the use of these substances in patients who lack other therapeutic options.

\section{BRAF}

Activating BRAF V600E mutations are found in 3-5\% of patients with CCA [27]. In contrast to melanoma, no convincing efficacy could be achieved in GI tumors with anti-BRAF directed monotherapy [41]. However, recent data support the sequential inhibition of the EGFR pathway using a combination of EGFR and BRAF or BRAF and MEK inhibitors. In CCA, co-treatment with BRAF and MEK inhibitors yielded highly promising results.

The ROAR basket trial included 43 partly intensively pretreated patients with $\mathrm{BRAF}^{\mathrm{V} 600 \mathrm{E}}$ mutations. The combination of trametinib (MEK inhibitor) and dabrafenib (BRAF inhibitor) achieved a convincing ORR of $51 \%$ (95\% CI 36-67, investigator-assessed) with a clinically significant mPFS of 9 months (95\% CI 5-10) and a mOS of 14 months (95\% CI 10-33). Similar positive signals were also reported from the NCI Match study, likewise a precision medicine basket trial, in which 3 out of 4 patients with BRAF V600E mutant biliary tumors treated 
with trametinib/dabrafenib achieved a partial response. We believe that these data highlight the benefits of molecular targeted therapy in patients with biliary tumors, and that BTC patients should be screened for the presence of BRAF mutations.

\section{NTRK}

Additional rare targetable events include fusions of the neurotrophic tyrosine kinase receptor (NTRK) genes that code for the tropomyosin receptor kinase receptors. The NAVIGATE phase II study included patients suffering from one of 17 different NTRK fusion-positive cancers, but only two patients with CCA [42]. In the overall population, the NTRK inhibitor larotrectinib achieved an impressive ORR of $80 \%$ with long-lasting tumor control. These results led to a tumor agnostic approval of the substance. A major challenge in the identification of NTRK fusionpositive cases is their very low prevalence. Due to the high therapeutic relevance and the tumor agnostic approval of larotrectinib and entrectinib, patients should be screened for NTRK fusion at least by immunohistochemistry.

\section{Immunotherapy}

Immunotherapeutic approaches are also under active investigation in patients with BTC. In contrast to the promising results in hepatocellular carcinoma, which have recently been confirmed in phase III studies, no convincing data are available yet for biliary tumors. In an early study that involved 24 patients with PD-L1-positive CCAs, the response rates of $20 \%$ in patients who received the PD-1 antibody pembrolizumab was comparable to other solid tumors [11]. However, Keynote-158, the largest immune-oncology study in CCA to date, reported disappointing results with a low response rate of 5.8\% [43]. These initial data suggest that clinically significant benefits can only be achieved in a small subset of patients with advanced BTC. As expected, high response rates were observed for pembrolizumab in BTC with microsatellite instability (MSI); however, MSI-high tumors account for less than $2 \%$ of the patient population [44].

For patients with microsatellite-stable tumors, combination therapies may be more effective. A first analysis for the combination of GemCis with the PD-L1 antibody durvalumab and with and without the CTLA- 4 antibody tremelimumab was presented at ASCO 2020. In this Korean patient population, the combination of dual checkpoint inhibition with GemCis demonstrated a very promising efficacy with a DCR of $98 \%$ and a mPFS of 11.9 (95\% CI 10.1-13.7) and a mOS of 20.7 (95\% CI 13.8-27.6) months. Interestingly, the tumor mutational burden did not correlate with mPFS or mOS. Whether these data can be confirmed in Caucasian patients needs to be deter- mined in ongoing studies such as TOPAZ-1 and KEYNOTE-966, in which patients are treated with GemCis alone or in combination with durvalumab or pembrolizumab. Overall, however, there are no sufficiently convincing data thus far that justify the use of immunotherapy outside of clinical trials in microsatellite-stable BTC.

\section{Conclusion}

After several years without significant progress in the treatment of BTC, a number of important studies have been successfully completed or initiated in the field that will generate meaningful insights for future systemic therapy concepts. Already today, for the first time, evidence-based approaches can be offered in the second line. The importance of immunotherapy is subject to ongoing investigation and should, at present, only be recommended to MSI patients.

A better understanding of the mutational spectrum of BTC has not only helped to realize the promise of targeted therapies in these cancers, but also to recognize the fundamental importance of patient stratification. In order to ensure that valid therapeutic options are not withheld from BTC patients, molecular testing should be performed at an early stage in the palliative setting and include MSI, BRAF and IDH1/2 mutations, FGFR2 and NTRK fusions, HER2/neu amplifications, and mutations. Several treatment decisions, especially in second and higher line, will remain individualized approaches, and we recommend that BTC patients are treated in specialized centers with a long-standing expertise in hepatobiliary surgery, loco-regional therapies, as well as systemic therapies.

\section{Conflict of Interest Statement}

Honoraria for Speaker, consultancy and advisory role to A.V.: Roche, Bayer, Sanofi, BMS, Lilly, Novartis, EISAI, AstraZeneca, Merck, Medac, Ipsen, PierreFabre, MSD. Honoraria for Speaker, consultancy and advisory role to A.S.: none.

\section{Funding Sources}

A.S. and A.V. have received funding from the Deutsche Forschungsgemeinschaft (DFG, German Research Foundation) - SFB/ TRR 209 - 314905040, and Vo959/9-1 (to A.V.). A.V. is supported by the European-Latin- American ESCALON consortium, funded by the EU Horizon 2020 program. A.S. is supported by Deutsche Krebshilfe (DK) - 70114101.

\section{Author Contributions}

Writing: both authors contributed equally. 


\section{References}

1 Banales JM, Marin JJ, Lamarca A, Rodrigues PM, Khan SA, Roberts LR, et al. Cholangiocarcinoma 2020: the next horizon in mechanisms and management. Nat Rev Gastroenterol Hepatol. 2020 Sep;17(9):557-88.

2 Valle JW, Bai LY, Orlova R, Cutsem EV, Alfonso JA, Chen LT, et al. Ramucirumab (RAM) or merestinib (MER) or placebo (PL) plus gemcitabine (GEM) and cisplatin (CIS) as first-line treatment for advanced or metastatic biliary tract cancer (BTC): A randomized, double-blind, phase II study. J Clin Oncol. 2020;38(4 suppl):477.

3 Valle J, Wasan H, Palmer DH, Cunningham D, Anthoney A, Maraveyas A, et al.; ABC-02 Trial Investigators. Cisplatin plus gemcitabine versus gemcitabine for biliary tract cancer. N Engl J Med. 2010 Apr;362(14): 1273-81.

4 Mavros MN, Economopoulos KP, Alexiou VG, Pawlik TM. Treatment and Prognosis for Patients With Intrahepatic Cholangiocarcinoma: Systematic Review and Meta-analysis. JAMA Surg. 2014 Jun;149(6):565-74.

5 Tsilimigras DI, Sahara K, Wu L, Moris D, Bagante F, Guglielmi A, et al. Very Early Recurrence After Liver Resection for Intrahepatic Cholangiocarcinoma: Considering $\mathrm{Al}$ ternative Treatment Approaches. JAMA Surg. 2020 Sep;155(9):823-31.

6 Horgan AM, Amir E, Walter T, Knox JJ. Adjuvant therapy in the treatment of biliary tract cancer: a systematic review and meta-analysis. J Clin Oncol. 2012 Jun;30(16):1934-40.

7 Tran Cao HS, Zhang Q, Sada YH, Chai C, Curley SA, Massarweh NN. The role of surgery and adjuvant therapy in lymph nodepositive cancers of the gallbladder and intrahepatic bile ducts. Cancer. 2018 Jan;124(1): 74-83.

8 Schweitzer N, Weber T, Kirstein MM, Fischer M, Kratzel AM, Reineke-Plaaß T, et al. The effect of adjuvant chemotherapy in patients with intrahepatic cholangiocarcinoma: a matched pair analysis. J Cancer Res Clin Oncol. 2017 Jul;143(7):1347-55.

9 Edeline J, Bonnetain F, Phelip JM, Watelet J, Hammel P, Joly JP, et al. Gemox versus surveillance following surgery of localized biliary tract cancer: results of the PRODIGE 12-ACCORD 18 (UNICANCER GI) phase III trial. J Clin Oncol. 2017;35(4 suppl):225.

10 Ebata T, Hirano S, Konishi M, Uesaka K, Tsuchiya Y, Ohtsuka M, et al.; Bile Duct Cancer Adjuvant Trial (BCAT) Study Group. Randomized clinical trial of adjuvant gemcitabine chemotherapy versus observation in resected bile duct cancer. Br J Surg. 2018 Feb; 105(3):192-202.

11 Primrose JN, Fox RP, Palmer DH, Malik HZ, Prasad R, Mirza D, et al.; BILCAP study group. Capecitabine compared with observation in resected biliary tract cancer (BILCAP): a randomised, controlled, multicentre, phase 3 study. Lancet Oncol. 2019 May;20(5):66373.

12 Okusaka T, Nakachi K, Fukutomi A, Mizuno N, Ohkawa S, Funakoshi A, et al. Gemcitabine alone or in combination with cisplatin in patients with biliary tract cancer: a comparative multicentre study in Japan. Br J Cancer. 2010 Aug;103(4):469-74.

13 Valle JW, Furuse J, Jitlal M, Beare S, Mizuno $\mathrm{N}$, Wasan $\mathrm{H}$, et al. Cisplatin and gemcitabine for advanced biliary tract cancer: a meta-analysis of two randomised trials. Ann Oncol. 2014 Feb;25(2):391-8.

14 Malka D, Cervera P, Foulon S, Trarbach T, de la Fouchardière C, Boucher E, et al.; BINGO investigators. Gemcitabine and oxaliplatin with or without cetuximab in advanced biliary-tract cancer (BINGO): a randomised, open-label, non-comparative phase 2 trial. Lancet Oncol. 2014 Jul;15(8):819-28.

15 Kim ST, Kang JH, Lee J, Lee HW, Oh SY, Jang JS, et al. Capecitabine plus oxaliplatin versus gemcitabine plus oxaliplatin as first-line therapy for advanced biliary tract cancers: a multicenter, open-label, randomized, phase III, noninferiority trial. Ann Oncol. 2019 May 1; 30(5):788-95.

16 Sahai V, Catalano PJ, Zalupski MM, Lubner SJ, Menge MR, Nimeiri HS, et al. Nab-Paclitaxel and Gemcitabine as First-line Treatment of Advanced or Metastatic Cholangiocarcinoma: A Phase 2 Clinical Trial. JAMA Oncol. 2018 Dec;4(12):1707-12.

17 Knox JJ, McNamara MG, Goyal L, Doherty M, Springfeld C, Park JO, et al. NUC-1031 in combination with cisplatin for first-line treatment of patients with advanced biliary tract cancer (NuTide:121) [-TPS.]. J Clin Oncol. 2020;38(4 suppl):TPS602. https://doi. org/10.1200/JCO.2020.38.4_suppl.TPS602.

18 Shroff RT, Javle MM, Xiao L, Kaseb AO, Varadhachary GR, Wolff RA, et al. Gemcitabine, Cisplatin, and nab-Paclitaxel for the Treatment of Advanced Biliary Tract Cancers: A Phase 2 Clinical Trial. JAMA Oncol. 2019 Jun;5(6):824-30.

19 Phelip JM, Desrame J, Edeline J, Barbier E, Terrebonne E, Michel P, et al. Modified FOLFIRINOX versus CISGEM as first-line chemotherapy for advanced biliary tract cancer: results of AMEBICA PRODIGE 38 randomized phase II trial. Ann Oncol. 2020;31:S2601.

20 Lamarca A, Ross P, Wasan HS, Hubner RA, McNamara MG, Lopes A, et al. Advanced Intrahepatic Cholangiocarcinoma: Post Hoc Analysis of the ABC-01, -02, and -03 Clinical Trials. J Natl Cancer Inst. 2020 Feb;112(2): 200-10.

21 Lamarca A, Hubner RA, David Ryder W, Valle JW. Second-line chemotherapy in advanced biliary cancer: a systematic review. Ann Oncol. 2014 Dec;25(12):2328-38.

22 Belkouz A, de Vos-Geelen J, Mathôt RA, Eskens FA, van Gulik TM, van Oijen MG, et al. Efficacy and safety of FOLFIRINOX as salvage treatment in advanced biliary tract cancer: an open-label, single arm, phase 2 trial. $\mathrm{Br}$ J Cancer. 2020 Mar;122(5):634-9.

23 Valle JW, Wasan H, Lopes A, Backen AC, Palmer DH, Morris K, et al. Cediranib or placebo in combination with cisplatin and gemcitabine chemotherapy for patients with advanced biliary tract cancer (ABC-03): a randomised phase 2 trial. Lancet Oncol. 2015 Aug;16(8):967-78
24 Vogel A, Kasper S, Bitzer M, Block A, Sinn $\mathrm{M}$, Schulze-Bergkamen $\mathrm{H}$, et al. PICCA study: panitumumab in combination with cisplatin/gemcitabine chemotherapy in KRAS wild-type patients with biliary cancer-a randomised biomarker-driven clinical phase II AIO study. Eur J Cancer. 2018 Mar; 92:11-9.

25 Jusakul A, Cutcutache I, Yong CH, Lim JQ, Huang MN, Padmanabhan N, et al. WholeGenome and Epigenomic Landscapes of Etiologically Distinct Subtypes of Cholangiocarcinoma. Cancer Discov. 2017 Oct;7(10):111635.

26 Javle MM, Murugesan K, Shroff RT, Borad MJ, Abdel-Wahab R, Schrock AB, et al. Profiling of 3,634 cholangiocarcinomas (CCA) to identify genomic alterations (GA), tumor mutational burden (TMB), and genomic loss of heterozygosity (gLOH). J Clin Oncol. 2019; 37(15 suppl):4087.

27 Silverman IM, Murugesan K, Lihou CF, Féliz L, Frampton GM, Newton RC, et al. Comprehensive genomic profiling in FIGHT-202 reveals the landscape of actionable alterations in advanced cholangiocarcinoma. J Clin Oncol. 2019;37(15 suppl):4080.

28 Lowery MA, Abou-Alfa GK, Valle JW, Kelley RK, Goyal L, Shroff RT, et al. ClarIDHy: A phase 3, multicenter, randomized, doubleblind study of AG-120 vs placebo in patients with an advanced cholangiocarcinoma with an IDH1 mutation [-TPS.]. J Clin Oncol. 2017;35(15 suppl):TPS4142.

29 Wu YM, Su F, Kalyana-Sundaram S, Khazanov N, Ateeq B, Cao X, et al. Identification of targetable FGFR gene fusions in diverse cancers. Cancer Discov. 2013 Jun;3(6):63647.

30 Javle M, Lowery M, Shroff RT, Weiss KH, Springfeld C, Borad MJ, et al. Phase II Study of BGJ398 in Patients With FGFR-Altered Advanced Cholangiocarcinoma. J Clin Oncol. 2018 Jan;36(3):276-82.

31 Mazzaferro V, El-Rayes BF, Droz Dit Busset M, Cotsoglou C, Harris WP, Damjanov N, et al. Derazantinib (ARQ 087) in advanced or inoperable FGFR2 gene fusion-positive intrahepatic cholangiocarcinoma. Br J Cancer. 2019 Jan;120(2):165-71.

32 Abou-Alfa GK, Sahai V, Hollebecque A, Vaccaro G, Melisi D, Al-Rajabi R, et al. Pemigatinib for previously treated, locally advanced or metastatic cholangiocarcinoma: a multicentre, open-label, phase 2 study. Lancet Oncol. 2020 May;21(5):671-84.

33 Javle MM, Borbath I, Clarke SJ, Hitre E, Louvet $C$, Mercade TM, et al. Infigratinib versus gemcitabine plus cisplatin multicenter, openlabel, randomized, phase 3 study in patients with advanced cholangiocarcinoma with FGFR2 gene fusions/translocations: the PROOF trial [-TPS.]. J Clin Oncol. 2019; 37(15 suppl):TPS4155.

34 Bekaii-Saab TS, Valle JW, Cutsem EV, Rimassa L, Furuse J, Ioka T, et al. FIGHT-302: firstline pemigatinib vs gemcitabine plus cisplatin for advanced cholangiocarcinoma with FGFR2 rearrangements. Future Oncol. 2020 Oct;16(30):2385-99. 
35 Yan M, Schwaederle M, Arguello D, Millis SZ, Gatalica Z, Kurzrock R. HER2 expression status in diverse cancers: review of results from 37,992 patients. Cancer Metastasis Rev. 2015 Mar;34(1):157-64.

36 Roa I, de Toro G, Schalper K, de Aretxabala $\mathrm{X}$, Churi C, Javle M. Overexpression of the HER2/neu Gene: A New Therapeutic Possibility for Patients With Advanced Gallbladder Cancer. Gastrointest Cancer Res. 2014 Mar; 7(2):42-8.

37 Li M, Zhang Z, Li X, Ye J, Wu X, Tan Z, et al. Whole-exome and targeted gene sequencing of gallbladder carcinoma identifies recurrent mutations in the ErbB pathway. Nat Genet. 2014 Aug;46(8):872-6.

38 Javle MM, Hainsworth JD, Swanton C, Burris HA, Kurzrock R, Sweeney C, et al. Pertuzumab + trastuzumab for HER2-positive metastatic biliary cancer: preliminary data from MyPathway. J Clin Oncol. 2017;35(4 suppl):402.
39 Hainsworth JD, Meric-Bernstam F, Swanton C, Hurwitz H, Spigel DR, Sweeney C, et al. Targeted Therapy for Advanced Solid Tumors on the Basis of Molecular Profiles: Results From MyPathway, an Open-Label, Phase IIa Multiple Basket Study. J Clin Oncol. 2018 Feb;36(6):536-42.

40 Harding J, Cleary J, Shapiro G, Braña I, Moreno V, Quinn D, et al. Treating HER2-mutant advanced biliary tract cancer with neratinib: benefits of HER2-directed targeted therapy in the phase 2 SUMMIT 'basket' trial. Ann Oncol. 2019;30(Supplement_4):IV127.

41 Hyman DM, Puzanov I, Subbiah V, Faris JE, Chau I, Blay JY, et al. Vemurafenib in Multiple Nonmelanoma Cancers with BRAF V600 Mutations. N Engl J Med. 2015 Aug;373(8): 726-36.
42 Drilon A, Laetsch TW, Kummar S, DuBois SG, Lassen UN, Demetri GD, et al. Efficacy of Larotrectinib in TRK Fusion-Positive Cancers in Adults and Children. N Engl J Med. 2018 Feb;378(8):731-9.

43 Piha-Paul SA, Oh DY, Ueno M, Malka D, Chung HC, Nagrial A, et al. Efficacy and safety of pembrolizumab for the treatment of advanced biliary cancer: results from the KEYNOTE-158 and KEYNOTE-028 studies. Int J Cancer. 2020 Oct;147(8):2190-8.

44 Marabelle A, Le DT, Ascierto PA, Di Giacomo AM, De Jesus-Acosta A, Delord JP, et al. Efficacy of Pembrolizumab in Patients With Noncolorectal High Microsatellite Instability/Mismatch Repair-Deficient Cancer: Results From the Phase II KEYNOTE-158 Study. J Clin Oncol. 2020 Jan;38(1):1-10. 